\title{
Fee Schedule Design when Debt Collection is Price Sensitive
}

\author{
Andres G. Abad, Ph.D. ${ }^{1}$, and Cinthia C. Pérez, Ph.D. ${ }^{1}$ \\ ${ }^{1}$ Escuela Superior Politécnica del Litoral (ESPOL), Guayaquil, Ecuador, agabad@espol.edu.ec, ccperez@espol.edu.ec
}

Abstract- When payment for a good (or service) is not required to be done immediately after its delivery, collection in full may not be possible. This is critical for post-paid billing systems such as mobile phone, water, electricity, and other utility service providers. In order to maximize their revenue, firms of these characteristics must not focus solely on increasing sales; instead, more importantly, they should focus on increasing collection. Thus, pricing should consider not only the good's demand elasticity, but also market's payment capacity-or its willingness to pay. We propose a linear programming model that can be used to maximize a firm's revenue collection over a one-period decision horizon. Our model works by segmenting customers based on their consumption level and assumes that customer's willingness to pay is similar within each segment. Prices are, then, found for each customers' segment. A case study is provided and its solution analyzed to develop further insights about the model.

Keywords - Pricing, willingness to pay, collected revenue

Digital Object Identifier (DOI): http://dx.doi.org/10.18687/LACCEI2015.1.1.244

ISBN: 13 978-0-9822896-8-6

ISSN: $2414-6668$

13 ${ }^{\text {th }}$ LACCEI Annual International Conference: “Engineering Education Facing the Grand Challenges, What Are We Doing?” July 29-31, 2015, Santo Domingo, Dominican Republic

DOI: $\underline{\text { http://dx.doi.org/10.18687/LACCEI2015.1.1.244 }}$ 


\title{
Fee Schedule Design when Debt Collection is Price Sensitive
}

\author{
Andres G. Abad, Ph.D. ${ }^{1}$, and Cinthia C. Pérez, Ph.D. ${ }^{1}$ \\ ${ }^{1}$ Escuela Superior Politécnica del Litoral (ESPOL), Guayaquil, Ecuador, \\ agabad@espol.edu.ec, ccperez@espol.edu.ec
}

\begin{abstract}
When payment for a good (or service) is not required to be done inmediately after its delivery, collection in full may not be possible. This is critical for post-paid billing systems such as mobile phone, water, electricity, and other utility service providers. In order to maximize their revenue, firms of these characteristics must not focus solely on increasing sales; instead, more importantly, they should focus on increasing collection. Thus, pricing should consider not only the good's demand elasticity, but also market's payment capacity—or its willingness to pay. We propose a linear programming model that can be used to maximize a firm's revenue collection over a one-period decision horizon. Our model works by segmenting customers based on their consumption level and assumes that customer's willingness to pay is similar within each segment. Prices are, then, found for each customers' segment. A case study is provided and its solution analyzed to develop further insights about the model.
\end{abstract}

Keywords-Pricing, willingness to pay, collected revenue.

\section{INTRODUCTION AND LITERATURE REVIEW}

When payment for a good (or service) is not done immediately after its delivery, it may not be possible to fully collect the debt. According to Zeng et al. in [1] the two main factors for invoice-payment risk are customer's financial capabilities and their willingness to pay. In their work, willingness to pay is defined as the maximum amount that someone is willing to pay to purchase a good or service.

Under these circumstances if a firm wants to find a price for a good or service that maximizes its revenue, the pricing model should not focus solely on increasing demand. The reason for this is that selling a good or service may not result in revenue if the customer decides not to pay the bill. The previous observation becomes more critical when the billing process follows a post-paid billing system, as in the case of mobile phone, water, electricity, and other utility service providers.

Moreover, the behavior of customers for post-paid goods is not exclusively related to the per unit fee, as in a regular price elasticity demand relationship. This has been confirmed empirically in the work presented by Inglesi-Lotz and Blignaut in [2], where a panel data study was conducted to assess electricity price elasticity in different sectors. The study concludes that the industrial sector was the only sector showing price elasticity for electricity; in sectors such as agriculture, transportation, and mining, the price of electricity did not affect its consumption.

The effect of price for post-paid goods or services can be understood by noting that the purchase decision and payment decisions are taken at different moments in the acquisition process, as shown in Figure 1. In pre-paid billing systems, a customer decides to purchase a good just after the price is known, hence that decision is directly affected by price elasticity of demand. On the other hand, in post-paid billing systems the customer decides to pay just after the good has been consumed, and the bill received. As a result, the decision to disburse is affected by the customer's willingness to pay. Our model aims to define a price that increases a firm's collected revenue, therefore, focusing on the payment decision made by the customer.

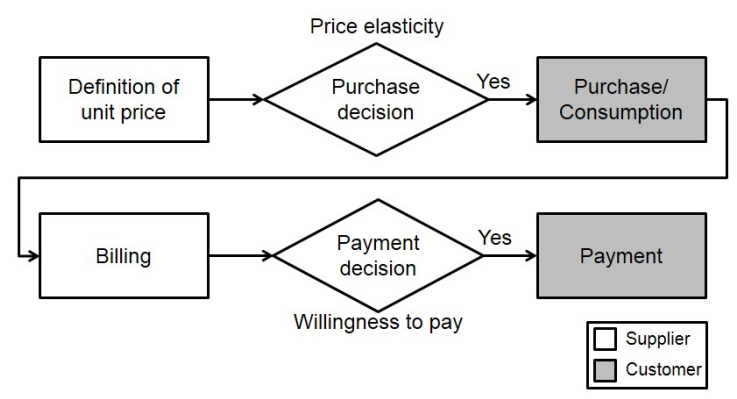

Fig. 1: Acquisition process for post-paid goods.

The effect of trade credit within a supply chain has been study by Fabbri and Klapper in [3], where they offer insights of how market power and financial constraints affects a firm's financial decisions and credit conditions for its customers. However, the effect of debt delinquency was not studied.

Credit policies have also been included in lot-sizing models, like in the one presented by Shinn in [4], where the retailers profit depends on the product price and credit terms offered by the seller. Abad and Jaggi in [5] presented a model where the unit price and the credit length are the decision variables, and analyze the model under the assumption of cooperative and non-cooperative structures. Chang et al. in [6] included a permissible delay in payment based on the order quantity in their lot-sizing model, where the market demand was price sensitive.

The previous models assume that payment will occur in a specified period of time. However, in practice, the payment may never occur, and therefore the actual profit is reduced.

We propose a one-period pricing model that can be used to maximize a firm's revenue, assuming that the firm offers a post-paid billing system to its customers. As in practical scenarios, customers may decide to pay the entire amount billed or only a portion of it. This decision depends on the total indebted amount and customer's financial capability. In 
this paper, we are not including the consequences of debt delinquency for customers-such as, service interruption or late payment fees-since we are only focusing on the firm's revenue during one period.

Our model can be used, not only within commercial environments, but also from a social perspective. Policy makers interested in defining the price for social goods or services, may offer price differentiation by taking into account the willingness to pay of social sectors that can only assume low debt levels. As a result, the model can maximize the actual revenue, and reduce the negative impact of outstanding debt and service disruption.

The remainder of this paper is organized as follows. Section II describes the main assumptions about customer's consumption and payment behavior. Section III presents the pricing model, where the revenue function is maximized. Section IV shows numerical results obtained from the model implementation, and Section V summarizes our work and discusses future research directions.

\section{Customer's Consumption And Payment DESCRIPTION}

In this section we give a mathematical description of customer's consumption and payment behavior for a particular good (or service). In what follows we will use the term product implying, either, good or service.

The quantity $q$ consumed of a product is considered as a random variable with a probability distribution $F_{q}$, and expectation defined as $E[q]=\mu$.

We define $f(q)$ as the fee charged for the $q^{\text {th }}$ unit consumed. Fee $f(q)$ may be affected by internal or external factors. For example, when the consumption of a good needs to be regulated, $f(q)$ can be increasing in $q$. On the other hand, if economies of scale are present, then $f(q)$ can be decreasing in $q$.

Fee $f(q)$ is not necessarily a continuous function. Particularly, in this paper we are interested in defining a fee schedule, where $f(q)$ is defined as a stepwise function and customers are grouped into subsets $R_{m}$ 's, for $m \in \mathcal{M}=\{1, \ldots, M\}$. In order to create the groups, we define breakpoints $r_{m} \in \mathbb{R}^{+}$, where $r_{m-1}<r_{m}$. A client consuming $r_{m-1}<q \leq r_{m}$ is then assigned to subset $R_{m}$. In Appendix $\mathrm{A}$ we show a procedure for obtaining breakpoints $r_{m}$ from data. Figure 2 shows an example of such a fee schedule.

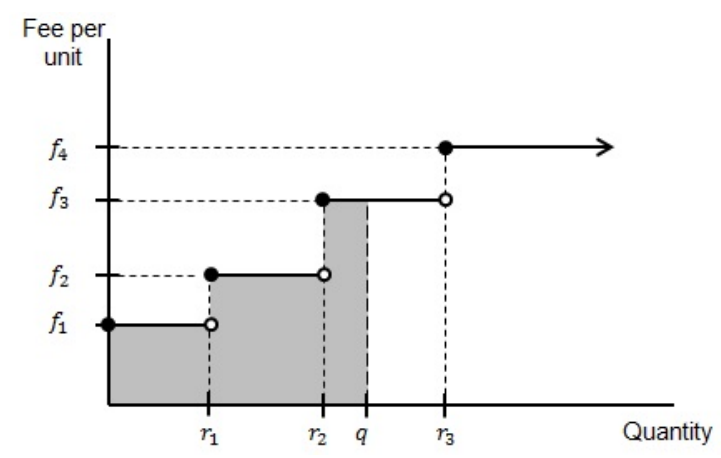

Fig. 2: Stepwise fee function.
The total amount billed to a customer consuming $q$ units is thus given by

$$
B(q)=\left(q-r_{m-1}\right) f_{m}+\sum_{j=1}^{m-1}\left(r_{j}-r_{j-1}\right) f_{j},
$$

for $q \in R_{m}$. In Figure 2, the shaded area corresponds to the total amount billed $B(q)$, for $q \in R_{3}$.

We assume that the amount paid by a customer depends on the amount billed $B(q)$. The proportion of the bill payed is a random quantity $\lambda_{q} \in[0,1]$, with expectation $E\left[\lambda_{q}\right]=\pi_{q}$. Note that $\pi_{q}$ depends on $q$.

The collected amount $C_{q}$ for a consumption of $q$ units is, thus, given by

$$
C_{q}=B(q) \lambda_{q}
$$

where $0 \leq C_{q} \leq B(q)$. Due to its construction, note that $C_{q}$ is a random variable.

For a particular value of $q$, the expected collected amount $E\left[C_{q} \mid q\right]$ is given by

$$
E\left[C_{q} \mid q\right]=B(q) \pi_{q}
$$

Assuming that there are $N$ customers whose consumption levels are independent, we may express the set of individual consumptions $q_{n}$ for $n \in \mathcal{N}=\{1, \ldots, N\}$ as an i.i.d. random sample $Q_{N}=\left\{q_{1}, \ldots, q_{N}\right\}$, where $q_{n} \sim F_{q}$.

The total amount collected from all customers within a sample $Q$, defined as $T(Q)$, is given by

$$
\begin{aligned}
T(Q) & =\sum_{q \in Q} C_{q} \\
& =\sum_{q \in Q} B(q) \lambda_{q} .
\end{aligned}
$$

We may compute the expectation of the total amount collected, given a random sample $Q_{N}, E\left[T(Q) \mid Q=Q_{N}\right]$, as

$$
\begin{aligned}
E\left[T(Q) \mid Q=Q_{N}\right] & =\sum_{n \in \mathcal{N}} E\left[C_{q} \mid q=q_{n}\right] \\
& =\sum_{n \in \mathcal{N}} B\left(q_{n}\right) E\left[\lambda_{q_{n}}\right] \\
& =\sum_{n \in \mathcal{N}} B\left(q_{n}\right) \pi_{q_{n}} .
\end{aligned}
$$

In this paper, we are interested in defining a fee schedule model that maximizes the expected total amount collected $E\left[T(Q) \mid Q=Q_{N}\right]$, assuming that the individual consumptions are known. The expression for the unconditional expectation $E[T(Q)]$ when the individual consumptions are unknown is discussed in Appendix B.

\section{FeE SCHEdule Model}

In this section we present a linear programming model for the design of a fee schedule that maximizes our expected total amount collected and that includes a price differentiation based on volume consumed. The model considers a product with inelastic demand.

In our model we will consider that the product fee is increasing in the volume consumed. This can be the case for 
scarce products, when the supplier is interested in moderating customer's consumption. Note, however, that if a supplier is willing to offer price reduction due to economies of scale, our model can be easily modified to consider such decreasing fees.

We want to maximize the total amount billed, denoted by $z=E\left[T(Q) \mid Q=Q_{N}\right]$. With this in mind, we define our linear programming model as

$$
\begin{gathered}
\max _{f_{m}} z \\
\text { s.t. } \\
z \geq K+k \sum_{q \in Q_{N}} q, \\
\sum_{q \in R_{m}} B(q) \pi_{q} \geq z \cdot \alpha_{m}, \quad \text { for } m \in \mathcal{M} \\
f_{m}-f_{m-1} \geq \delta_{L}^{m}, \quad \text { for } m \in \mathcal{M} \\
f_{m}-f_{m-1} \leq \delta_{U}^{m}, \quad \text { for } m \in \mathcal{M} \\
f_{m} \in \mathbb{R}^{+}, \quad \text { for } m \in \mathcal{M}
\end{gathered}
$$

Here the parameters are:

$N \quad$ Number of customers

$q_{n} \quad$ Quantity of product consumed by customer $n \in$ $\mathcal{N}$. We assume that $q_{n} \leq q_{n+1}$ for $n \in \mathcal{N}$

$K \quad$ Fixed cost incurred by the supplier

$k \quad$ Variable cost per unit, incurred by the supplier

$M \quad$ Number of subsets of customers aggregated by consumption

$\alpha_{m} \quad$ Fraction of the total amount collected from customers in subset $R_{m}$ for $m \in \mathcal{M}$

$\delta_{m}^{u} \quad$ Upper bound for the difference in the fee between customers in subset $m-1$ and $m$, for $m \in \mathcal{M}$

$\delta_{m}^{l} \quad$ Lower bound for the difference in the fee between customers in subset $m-1$ and $m$, for $m \in \mathcal{M}$

$\pi_{q} \quad$ Expectation of the proportion of the bill payed by a customer that consumes $q$ units of product

$r_{m} \quad$ Break point defining subsets $R_{m}$, such that $q \in$ $R_{m}$ iff $r_{m-1}<q \leq r_{m}$, for $m \in \mathcal{M}$. We define $r_{0}=0$, and $r_{M}=\max _{n \in \mathcal{N}} q_{n}$,

and the variables are:

$f_{m} \quad$ Fee per unit of product consumed for customers with consumption level $q \in R_{m}$, where $m \in \mathcal{M}$. We define $f_{1} \geq \underline{f}$, and $f_{M} \leq \bar{f}$.

We want to maximize the revenue obtained by the supplier after debt collection, defined in Section II. The constraint defined in (1b) guarantees that the total amount collected covers the fixed and variables costs for the firm. Note that, if (1b) is not satisfied, the firm will have a deficit and may decide not to supply the product. Constraint (1c) makes sure that the percentage of the total revenue collected from each subset of customers is at least equal to $\alpha_{m}$. Note that $\alpha_{m}$ can be set equal to zero, when there is no lower bound in the revenue that has to be collected from a subset $R_{m}$. The set of constraints (1d) and (1e) guarantee that the increase in the product fee does not exceeds the upper and lower bound defined by the supplier. Finally (1f) define the model variables as continuous and non-negative.

\section{NUMERICAL TEST}

In this section we provide a numerical case study for designing the fee schedule of an electricity provider firm. This case study shows the implementation of the proposed model. Additionally, we provide an analysis of the case study and its solution to develop further insights about the model. The case study uses monte-carlo simulation to generate a dataset of monthly consumptions.

\section{A. Generating the Dataset}

We generate $N=1,000$ data points corresponding to individual electricity consumption $q_{n}$, given in $\mathrm{kWh}$. An exponential distribution was used to generate the amount consumed monthly, with mean value $289.25 \mathrm{kWh} /$ month.

The consumption range is subdivided into four subsets $R_{1}, \ldots, R_{4}$, as shown in Figure 3 . The first subset $R_{1}$ is defined to contain the bottom $40 \%$ of the customers in terms of their consumption. The second subset $R_{2}$, is defined to contain the next lowest $35 \%$ of the customers in terms of their consumption. Likewise, for $R_{3}$ and $R_{4}$ we used the next $20 \%$, and $5 \%$, respectively.

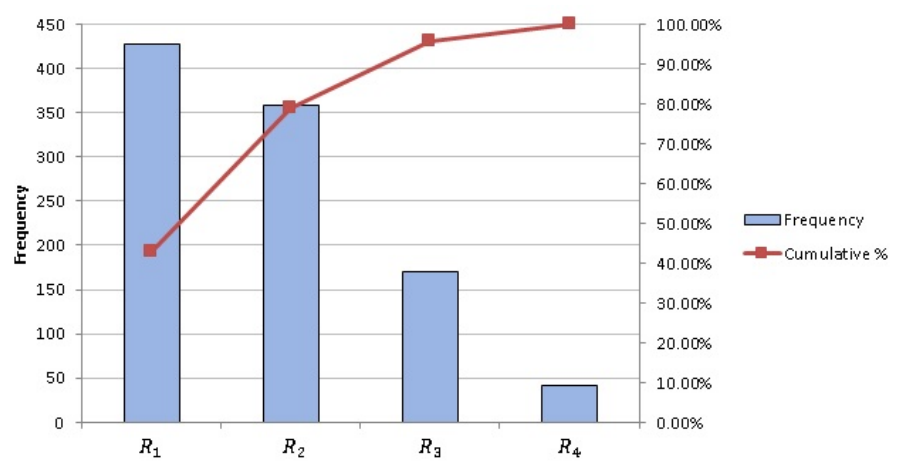

Fig. 3: Histogram of generated consumptions.

Points $r_{m}$ were defined analytically as the $0.4,0.75,0.95$, and 1 quantile of the exponential distribution, respectively. The resulting $r_{m}$ points, as well as the corresponding consumption within each subset $R_{m}$, are shown in Table I.

TABLE I: Generated consumption

\begin{tabular}{cccc}
\hline$m$ & $r_{m}$ & \# of customers in subset & Consumption within subset \\
\hline 1 & 153 & 429 & $30,387.39$ \\
2 & 416 & 359 & $93,524.05$ \\
3 & 899 & 170 & $99,804.07$ \\
4 & $\max _{i}\left\{q_{i}\right\}$ & 42 & $46,929.03$ \\
\hline
\end{tabular}

\section{B. Definition of Model Parameters}

In this section we present the parameters used in our numerical case study. The parameters used were chosen to emulate a realistic scenario.

For overhead costs $K$ we used $\$ 11,934.73$, and for variable costs $k$ we used $0.038 \$ / \mathrm{kWh}$. Using these parameters, the total 
cost for generation and distribution is obtained as $K+k \sum_{i} q_{i}$ which is $\$ 22,316.65$.

Table II shows the numerical values used for the difference of the fees $\delta_{m}^{u}$ and $\delta_{m}^{l}$, the fraction $\alpha_{m}$ of the total amount collected for customers in subset $R_{m}$, as well as the proportion of the bill payed $\pi_{m}$ for each customer in subset $R_{m}$.

TABLE II: Used parameters

\begin{tabular}{ccccc}
\hline$m$ & $\delta_{m}^{l}$ & $\delta_{m}^{u}$ & $\alpha_{m}$ & $\pi_{m}$ \\
\hline 1 & -2 & 2 & 0.2 & 0.4 \\
2 & -2 & 2 & 0.6 & 0.9 \\
3 & -2 & 2 & 0.5 & 0.8 \\
4 & -2 & 2 & 0.3 & 0.7 \\
\hline
\end{tabular}

We used an inverted "U" shape for the parameters $\pi_{m}$, as shown in Figure 4, assuming that customers in the low range of consumption will be more likely to have financial difficulties, and therefore, the proportion of the bill payed will be low; while customers in the high range of consumption defaults often.

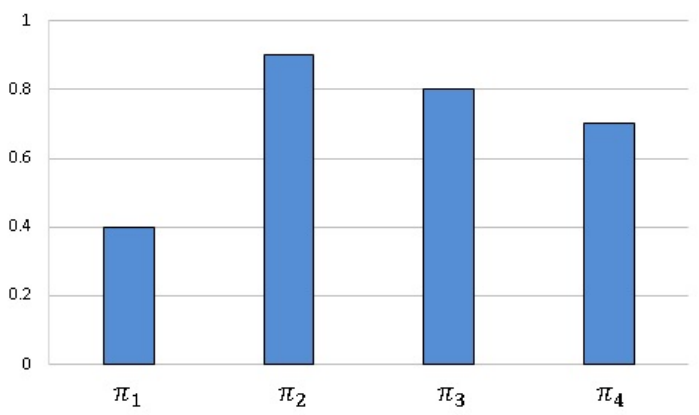

Fig. 4: Proportion $\pi_{m}$ of bill payed for each subset $R_{m}$.

\section{Results and Analysis}

The linear programming model, for these particular choice of parameters, proofs to be feasible and provides the fee schedule $f_{m}$, for $m=1, \ldots, 4$. The results are presented in Table III.

TABLE III: Results

\begin{tabular}{cccc}
\hline$m$ & $f_{m}$ & Total Billed for $R_{m}$ & Total collected from $R_{m}$ \\
\hline 1 & 1.08 & $\$ 32,818.38$ & $\$ 13,127.35$ \\
2 & 3.08 & $\$ 178,022.24$ & $\$ 160,220.02$ \\
3 & 2.00 & $\$ 223,861.02$ & $\$ 179,088.82$ \\
4 & 2.00 & $\$ 81,484.34$ & $\$ 57,039.03$ \\
\hline & Total & $\$ 516,185.99$ & $\$ 409,475.23$ \\
\hline
\end{tabular}

The total aggregated amount billed to all the customers was $\$ 516,185.99$. Because of the willingness to pay, only $\$ 409,475.23$ was collected. It can readily be checked that the amount collected, $\$ 409,475.23$, covers the generation and distributions costs $\$ 22,316.65$ by a large margin.

\section{Conclusions And Future Directions}

Pricing models often focus on the effect of price on the product demand, without taking into consideration the actual amount collected. The effect of debt delinquency becomes more critical under post-paid billing systems, where the customer may decide not to pay the bill in full, even after the product has been consumed.

We propose a pricing model that finds the optimal fee schedule to increase a firm's collected revenue. Our model assumes that customers are aggregated by consumption and bill payment behavior, and finds the optimal unit fee for each subset of customers.

By including information about the expected proportion of the bill payed by the customers, our model allows the seller to define fees that guarantee that amount collected will be at least equal to the total cost. Also, in a way, this model can ensures a fair fee definition, since subset of customers with known risk of debt default can be identified and controlled.

As future research, we would like to consider the values for the breakpoints $r_{m}$, that define the fee function, as model variables. In this way, the model will define how the customers will be aggregated. Also, we would like to extend our model to a finite number of periods, and therefore, include the collected amount from customers with late payments. A multi-period model will further allow us to evaluate the efficiency of our pricing decisions, making sure that initial investment costs and operational cost are covered over the considered time horizon.

\section{APPENDIX A \\ Estimation of the Proportion Payed By EACH CUSTOMER}

Here we will show a procedure to estimate the proportion payed $\pi_{q}$ of the total bill $B(q)$. The procedure assumes that we have a dataset containing historical billed amounts $B\left(q_{n}\right)$ and their corresponding collected amounts $C_{q_{n}}$, for $n \in \mathcal{N}$.

Our estimation approach seeks to minimize the divergence between observed and predicted values. To measure this divergence we use the sum of the absolute differences, and formulate the following optimization problem

$$
\begin{array}{lc}
\min _{\pi_{q_{n}}} & \sum_{q \in Q_{N}}\left|C_{q}-E\left[C_{q}\right]\right| \\
\text { s.t.: } & 0 \leq \pi_{q_{n}} \leq 1, \quad \text { for } n \in \mathcal{N},
\end{array}
$$

where $E\left[C_{q}\right]=B(q) \pi_{q}$.

This problem can be cast as a linear programming problem as

$$
\begin{aligned}
& \min _{\pi_{q_{n}}} \quad \sum_{n \in \mathcal{N}} \varepsilon_{n}^{+}+\varepsilon_{n}^{-} \\
& \text {s.t.: } \quad C_{q_{n}}-B\left(q_{n}\right) \pi_{q_{n}} \leq \varepsilon_{n}^{+}, \quad \text { for } n \in \mathcal{N} \\
& B\left(q_{n}\right) \pi_{q_{n}}-C_{q_{n}} \leq \varepsilon_{n}^{-}, \quad \text { for } n \in \mathcal{N} \\
& \pi_{q_{n}} \leq 1, \quad \text { for } n \in \mathcal{N} \\
& \pi_{q_{n}}, \varepsilon_{n}^{+}, \varepsilon_{n}^{-} \geq 0, \quad \text { for } n \in \mathcal{N} \text {, }
\end{aligned}
$$

and can be trivially solved by

$$
\pi_{q_{n}}=\frac{C_{q_{n}}}{B\left(q_{n}\right)}, \quad \text { for } n \in \mathcal{N} .
$$

Note that, since $C_{q_{n}} \leq B\left(q_{n}\right)$, then $0 \leq \pi_{q_{n}} \leq 1$.

However, having the value of $\pi_{q_{n}}$ for each customer $n$ with a specific consumption level $q_{n}$ is not useful when we are trying to define a general fee schedule. 
We now consider the setting in which customers have been classified into different subsets $R_{m}$, for $m \in \mathcal{M}$, depending on their amount consumed. If customers' willingness to pay is similar within each subset $R_{m}$, we may then assume that there is a single $\pi_{m}$ for clients in $R_{m}$. The resulting optimization problem is now

$$
\begin{aligned}
& \min _{\pi_{1} \ldots, \pi_{M}} \sum_{n \in \mathcal{N}} \varepsilon_{n}^{+}+\varepsilon_{n}^{-} \\
& \text {s.t.: } \\
& C_{q_{n}}-B\left(q_{n}\right) \pi_{m} \leq \varepsilon_{n}^{+} \text {, for } m \in \mathcal{M} \text {, and }\left\{n: q_{n} \in R_{m}\right\} \\
& B\left(q_{n}\right) \pi_{m}-C_{q_{n}} \leq \varepsilon_{n}^{-}, \text {for } m \in \mathcal{M} \text {, and }\left\{n: q_{n} \in R_{m}\right\} \\
& \pi_{m-1} r_{m-1}=\pi_{m} r_{m-1} \text {, for } m \in \mathcal{M} \backslash\{1\} \\
& \pi_{m} \leq 1, \text { for } m \in \mathcal{M} \\
& \pi_{m} \geq 0, \text { for } m \in \mathcal{M} \\
& \varepsilon_{n}^{+}, \varepsilon_{n}^{-} \geq 0 \text {, for } n \in \mathcal{N} \text {. }
\end{aligned}
$$

The problem above can be used to estimate the expected payed amount, $\pi_{m}$, for customer within each subset $R_{m}$, for $m \in \mathcal{M}$.

\section{APPENDIX B}

\section{UnCONDITIONAL EXPECTATION OF THE TOTAL AMOUNT}

\section{COLlECTED}

We denote a random sample containing the consumption of $N$ customers by $\Omega=\left\{q_{1} \ldots, q_{N}\right\} \sim F_{q}^{N}$. We find the unconditional expectation $E[T(\Omega)]$ as

$$
\begin{aligned}
E[T(\Omega)] & =E\left[E\left[T(\Omega) \mid \Omega=Q_{N}\right]\right] \\
& =\sum_{q \in Q_{N}} E\left[E\left[C_{q} \mid \Omega=Q_{N}\right]\right] \\
& =\sum_{q \in Q_{N}} E\left[E\left[B(q) \lambda_{q} \mid \Omega=Q_{N}\right]\right] \\
& =E\left[\sum_{q \in Q_{N}} B(q) \pi_{q} \mid \Omega=Q_{N}\right] \\
& =N\left[B\left(\mu_{q}\right) \pi_{\mu_{q}}\right]+N \cdot \operatorname{COV}\left[B(q), \pi_{q}\right] .
\end{aligned}
$$

We consider that there is a dependency between the amount billed to a customer and the proportion of that bill actually payed, i.e., $B(q)$ and $\pi_{q}$ are regarded as dependent random variables. In general, however, we are not able to attribute $C O V\left[B(q), \pi_{q}\right]$ a particular value, since the exact relationship between $B(q)$ and $\pi_{q}$ cannot be generalized. Furthermore, even if the value for $C O V\left[B(q), \pi_{q}\right]$ can be found, its value may be different among different regions. This is attributed to the non-monotonic shape of $\pi_{q}$ as the one shown in Figure 4.

\section{REFERENCES}

[1] S. Zeng, P. Melville, C. A. Lang, I. Boier-Martin, and C. Murphy, "Using predictive analysis to improve invoice-to-cash collection," in Proceedings of the 14th ACM SIGKDD international conference on Knowledge discovery and data mining. ACM, 2008, pp. 1043-1050.

[2] R. Inglesi-Lotz and J. N. Blignaut, "Estimating the price elasticity of demand for electricity by sector in south africa," South African Journal of Economic and Management Sciences, vol. 14, no. 4, pp. 449-465, 2011.

[3] D. Fabbri and L. F. Klapper, "Trade credit and the supply chain," 2009.

[4] S. Shinn, "Determining optimal retail price and lot size under day-terms supplier credit," Computers \& industrial engineering, vol. 33, no. 3, pp. 717-720, 1997.

[5] P. L. Abad and C. K. Jaggi, "A joint approach for setting unit price and the length of the credit period for a seller when end demand is price sensitive," International Journal of Production Economics, vol. 83, no. 2, pp. 115-122, 2003.

[6] H.-C. Chang, C.-H. Ho, L.-Y. Ouyang, and C.-H. Su, "The optimal pricing and ordering policy for an integrated inventory model when trade credit linked to order quantity," Applied Mathematical Modelling, vol. 33, no. 7, pp. 2978-2991, 2009.

\section{ACKNOWLEDGMENT}

The authors would like to thank to the anonymous reviewers for their helpful comments and useful suggestions. 